\title{
Sequence diversity and copy number variation of Mutator-like transposases in wheat
}

\author{
Nobuaki Asakura ${ }^{1}$, Shinya Yoshida ${ }^{2}$, Naoki Mori ${ }^{3}$, Ichiro Ohtsuka ${ }^{1}$ and Chiharu Nakamura ${ }^{3}$ \\ ${ }^{1}$ Laboratory of Biology, Faculty of Engineering, Kanagawa University, Yokohama, Japan. \\ ${ }^{2}$ Hyogo Institute of Agriculture, Forestry \& Fisheries, Kasai, Japan. \\ ${ }^{3}$ Laboratory of Plant Genetics, Graduate School of Agricultural Science, Kobe University, Kobe, Japan.
}

\begin{abstract}
Partial transposase-coding sequences of Mutator-like elements (MULEs) were isolated from a wild einkorn wheat, Triticum urartu, by degenerate PCR. The isolated sequences were classified into a MuDR or Class I clade and divided into two distinct subclasses (subclass I and subclass II). The average pair-wise identity between members of both subclasses was $58.8 \%$ at the nucleotide sequence level. Sequence diversity of subclass I was larger than that of subclass II. DNA gel blot analysis showed that subclass I was present as low copy number elements in the genomes of all Triticum and Aegilops accessions surveyed, while subclass II was present as high copy number elements. These two subclasses seemed uncapable of recognizing each other for transposition. The number of copies of subclass II elements was much higher in Aegilops with the S, $\mathrm{S}^{\prime}$ and D genomes and polyploid Triticum species than in diploid Triticum with the A genome, indicating that active transposition occurred in S, $S^{\prime}$ and D genomes before polyploidization. DNA gel blot analysis of six species selected from three subfamilies of Poaceae demonstrated that only the tribe Triticeae possessed both subclasses. These results suggest that the differentiation of these two subclasses occurred before or immediately after the establishment of the tribe Triticeae.
\end{abstract}

Key words: Mutator-like transposase, sequence diversity, copy number variation, Triticum, Aegilops.

Received: September 6, 2007; Accepted: December 14, 2007.

\section{Introduction}

The Mutator trait with a high frequency of forward mutations was first identified in a single maize line (Robertson 1978). Like the $A c / D s$ and Spm/dSpm systems, Mutator activity is regulated by the two-component system composed of two DNA-type transposable elements, $M u D R$ and $M u$ (Bennetzen 1996). $M u D R$ is an autonomous element, while $M u$ is a non-autonomous deletion derivative of MuDR. Mutator-like elements (MULEs) have been identified in diverse plant species including both monocots and dicots (Mao et al. 2000; Yu et al. 2000; Lisch et. al. 2001; Rossi et al. 2001). Transposases sharing a homologous domain with MURA (mudrA product) were also found in prokaryotes (Eisen et al. 1994) and a MULE named Hop has been recently isolated from the fungus, Fusarium oxysporum (Chalvet et al. 2003). It is thus apparent that Mutator composes a superfamily and is widespread.

Molecular features and transposition mechanisms of Mutator have been extensively studied (for reviews see

Send correspondence to Nobuaki Asakura. Laboratory of Biology, Faculty of Engineering, Kanagawa University, 221-8686 Rokkakubashi, Kanagawa-ku, Yokohama, Japan. E-mail: asakura@ kanagawa-u.ac.jp.
Lisch 2002; Walbot and Rudenko 2002). MuDR is 4.9-kbp long and possesses around 200-bp of terminal inverted repeats (TIRs). During insertion, a 9-bp duplication of the recipient DNA is generated. $M u D R$ carries two genes, mudrA and $m u d r B$. The former encodes the MURA transposase that catalyzes the excision of Mutator (Eisen et al. 1994; Benito and Walbot 1997) and the latter encodes the MURB protein that is proposed to be involved in the reinsertion of Mutator (Lisch et al. 1999; Raizada and Walbot 2000). However, the sequence corresponding to $m u d r B$ has only been identified in the genus Zea so far (Lisch et al. 1995; Walbot and Rudenko 2002). Transposition activity of Mutator was differentially regulated in somatic and germinal tissues (Lisch et al. 1995; Raizada et al. 2001). Mutator had a cut-and-paste mechanism in somatic tissues, while it appeared to transpose either by a gap-repair mechanism or by a semi-conservative and duplicative transposition mechanism in germinal tissues. Consequently, numerous copies of Mutator can be accumulated in a given genome.

Wheat consists of a series of species with different ploidy levels and their genome differentiation and evolutionary history through allopolyploidization are well known (Kihara and Tanaka 1970; Kimber and Sears 1983; Feldman et al. 1995). Wheat is therefore an informative 
material to study MULE dynamics related to genome differentiation and evolution through allopolyploidization. Partial sequences of MULE transposases were isolated by PCR from several grass species including common wheat (Lisch et al. 2001). Genome sequencing analysis led to the identification of a few MULEs in einkorn wheat (Yan et al. 2002), durum wheat (Wicker et al. 2003) and common wheat (Chantret et al. 2005), as well as in Aegilops tauschii (synonymous to Ae. squarrosa), a wild D genome donor species to common wheat (Li et al. 2004; Chantret et al. 2005). However, little is known about MULE dynamics in Triticum and Aegilops.

We previously identified and characterized MULEs in rice (Yoshida et al. 1998; Asakura et al. 2002). One of these rice MULEs, OsMu4-2, carried a transcriptionally active gene encoding a putative transposase with a significantly high identity to maize MURA. We now performed degenerate PCR to isolate partial sequences of MULE transposase from Triticum urartu, a donor of the A genome to two tetraploid wheat groups (emmer and timopheevi) (Tsunewaki and Nakamura 1995). A pair of primers was designed based on the sequences of MURA and a putative transposase of $O s M u 4-2$. The isolated sequences possessed a conserved transposase-coding domain and were divided into two subclasses with different sequence diversity. DNA gel blot analysis in Triticum and Aegilops species revealed marked copy number variation between both subclasses. Furthermore, the copy number of MULEs of the high copy number subclass greatly differed between diploid Triticum and Aegilops species. In addition, we studied the distribution of the two MULE subclasses in other grass species.

\section{Materials and Methods}

\section{Plant materials}

Wild einkorn wheat, Triticum urartu (accession no. KU199-2, Plant Germplasm Institute, Kyoto University, Japan, Table 1), was used as a donor of template DNA for degenerate PCR amplification of the conserved transposase-coding region of MULEs. Twenty-eight accessions of Triticum and Aegilops species, including KU199-2, were used for DNA gel blot analysis. Oryza sativa (rice) from the subfamily Ehrhartoideae, Secale cereale (rye), Hordeum vulgare (barley) and Avena sativa (oat) from the subfamily Pooideae, Zea mays (maize) and Sorghum bicolor (sorghum) from the subfamily Panicoideae, were also used for DNA gel blot analysis.

\section{Degenerate PCR and sequencing of the amplified DNA fragments}

Total DNA was extracted from young leaves of KU199-2 with DNeasy Plant Maxi Kit (QIAGEN, Hilden, Germany). Degenerate primers were designed after comparing amino acid sequences of the conserved transposase region of the maize MURA and a putative transposase of rice OsMu4-2 (Asakura et al. 2002). The nucleotide sequences of the forward and reverse primers were 5'-GAY GGICAYAAYTGGATG-3' and 5'-GTGATRWARTCRC AYTTDAT-3', respectively. Coding sequences corresponding to the region between MURA amino acid residues 350 through 484 were amplified. The PCR amplification was carried out in $50 \mu \mathrm{L}$ of a reaction mixture containing $2.5 \mathrm{U}$ of Taq polymerase, $1.0 \mu \mathrm{M}$ of primers, $50 \mathrm{ng}$ of total DNA, $0.2 \mathrm{mM}$ of each dNTP, $1.5 \mathrm{mM}$ of $\mathrm{MgCl}_{2}$ and reaction buffer. PCR was performed in a GeneAmp PCR 9700 (Applied Biosystems, Foster city, CA) as follows: a pre-denaturation step of $1 \mathrm{~min}$ at $94^{\circ} \mathrm{C} ; 30$ cycles of denaturation for $1 \mathrm{~min}$ at $94^{\circ} \mathrm{C}$, annealing for $2 \mathrm{~min}$ at $55^{\circ} \mathrm{C}$ and extension for $2 \mathrm{~min}$ at $72^{\circ} \mathrm{C}$; followed by a post-extension incubation for $3 \mathrm{~min}$ at $72{ }^{\circ} \mathrm{C}$. Amplified DNA fragments were cloned into pGem-T Easy vector (Promega, Madison, Wis.) and used in the transformation of Escherichia coli strain DH5 $\alpha$. In order to exclude clones with unexpected DNA fragments, the length of the inserted DNA fragments was examined by direct colony PCR followed by agarose-gel electrophoresis. The sequencing of the inserts was performed by the dideoxynucleotide chain-termination method. Individual sequences of putative MULE transposases were designated MoTU, according to the nomenclature system of Lisch et al. (2001). Mo is the prefix of maize $m u d r A$-like sequences. $T$ and $U$ are the initials of Triticum urartu.

\section{Sequence analyses}

Putative amino acid sequences deduced from the nucleotide sequences of MoTUs were analyzed using the NCBI conserved domain search (Marchler-Bauer and Bryant 2004) in order to confirm that they coded for MULE transposases. Homologous sequences from Triticum and Aegilops were searched in the TIGR Wheat Genome Database using BLASTn and tBLASTx (Altschul et al. 1997). Two other Mutator-related elements, Trap (Comelli et al. 1999) and Jittery (Xu et al. 2004), have been identified in maize. Mutator-like transposases were divided into four classes in sugarcane, rice and Arabidopsis (Rossi et al. 2004). Nucleotide sequences of the conserved transposase region from MuDR, Trap, Jittery and representative clones, TE165, TE109, TE266 and $T E 148$, from each of the four MULE classes of sugarcane were used as queries in the homology search. To characterize MoTUs, individual sequences were compared to the query sequences, sequences of MULEs found in the homology search and OsMu4-2. Multiple alignments of coding sequences (amino acid residues 385 to 477 of MURA) were carried out using CLUSTAL X ver. 1.83 (Tompson et al. 1997). Phylogenetic analysis was performed by the neighbor-joining (NJ) method (Saitou and Nei 1980) using MEGA ver. 3.1 (Kumar et al. 2004). 


\section{DNA gel blot analysis}

DNA gel blot analysis was carried out to study the distribution of MULE transposases related to MoTUs in Triticum and Aegilops species and the distribution of homologous sequences among six other grass species. Total DNA was extracted from young leaves of all accessions using the DNeasy Plant Maxi Kit (QIAGEN). Genomic DNA of wheat, rye, barley and oat $(10 \mu \mathrm{g})$, and of rice, maize and sorghum $(5 \mu \mathrm{g})$ was digested with Hind III. The digests were fractionated by electrophoresis through $0.8 \%$ agarose gels and transferred onto nylon membranes using the alkaline blotting method. Two clones, MoTU-12 and MoTU-32, were used as probes. Labeling, hybridization and signal detection were performed using the Gene Images RandomPrime Labelling and Detection System (Amersham Biosciences) according to the manufacturer's instructions with slight modifications. Prehybridization and hybridization were conducted for five and $18 \mathrm{~h}$, respectively, at $65^{\circ} \mathrm{C}$ in buffer containing $5 \mathrm{xSSC}, 0.1 \%$ SDS, $5 \%$ dextran sulfate and $5 \%$ blocking reagent. Membranes were washed twice for $25 \mathrm{~min}$ at $65{ }^{\circ} \mathrm{C}$ in a solution containing $1 \times$ XSC and $0.1 \%$ SDS, followed by two $25 \mathrm{~min}$ washes at $65{ }^{\circ} \mathrm{C}$ in $0.1 \mathrm{xSSC}$ and $0.1 \%$ SDS (high stringency). Signals were detected after membranes exposure to X-ray films for about one hour.

\section{Results}

\section{Isolation of MULE transposases coding sequences from Triticum urartu}

Degenerate PCR was carried out to isolate partial coding sequences of MULE transposases from $T$. urartu (KU199-2, Table 1). After cloning the amplified DNA fragments into plasmids, the length of each inserted sequence was measured by direct colony PCR followed by an

Table 1 - Triticum and Aegilops accessions used in DNA gel blot analysis.

\begin{tabular}{|c|c|c|c|}
\hline Species (genome constitution) & Accession number ${ }^{1)}$ or cultivar name & Abbreviation & Origin \\
\hline \multirow[t]{5}{*}{ Triticum urartu (AA) } & PI 428316 & urr1 & Iran \\
\hline & PI 487268 & urr2 & Syria \\
\hline & KU199-2 & urr3 & Armenia \\
\hline & PI 428270 & urr4 & Lebanon \\
\hline & PI 428184 & urr5 & Turkey \\
\hline \multirow[t]{5}{*}{ Triticum boeoticum (AA) } & KU101-3 & btc1 & Iran \\
\hline & KU103 & btc2 & Iran \\
\hline & PI 427999 & btc3 & Lebanon \\
\hline & PI 538723 & btc4 & Turkey \\
\hline & PI 272556 & btc5 & Hungary \\
\hline \multirow[t]{2}{*}{ Triticum monococcum (AA) } & PI 167611 & $\mathrm{mnc1}$ & Turkey \\
\hline & PI 277138 & mnc2 & - \\
\hline Triticum sinskajae (AA) & Dr. Konzak ${ }^{2)}$ & sns1 & - \\
\hline Aegilops speltoides (SS) & KU2-5 & spl1 & Turkey \\
\hline Aegilops sharonensis $\left(\mathrm{S}^{1} \mathrm{~S}^{1}\right)$ & KU5-2 & $\operatorname{shr} 1$ & Israel \\
\hline Aegilops longissima $\left(\mathrm{S}^{1} \mathrm{~S}^{1}\right)$ & KU4-1 & $\operatorname{lng} 1$ & Israel \\
\hline \multirow[t]{2}{*}{ Aegilops tauschii (DD) } & KU20-2 & tsc1 & - \\
\hline & KU2074 & $\operatorname{tsc} 2$ & Iran \\
\hline \multirow[t]{2}{*}{ Triticum dicoccoides (AABB) } & KU198 & ded 1 & Israel \\
\hline & KU8821C & $\operatorname{dcd} 2$ & Turkey \\
\hline Triticum dicoccum (AABB) & KU113 & $\mathrm{dcm} 1$ & - \\
\hline Triticum durum (AABB) & Langdon & drm1 & - \\
\hline Triticum araraticum (AAGG) & KU196-2 & arr1 & USSR \\
\hline Triticum timopheevi (AAGG) & KU107-1 & tmp1 & - \\
\hline \multirow[t]{2}{*}{ Triticum spelta (AABBDD) } & $\mathrm{KIBR}^{3)}$ & splt1 & - \\
\hline & $\mathrm{KIBR}^{3)}$ & splt2 & - \\
\hline \multirow[t]{2}{*}{ Triticum aestivum (AABBDD) } & Chinese Spring & ast1 & - \\
\hline & Norin 26 & ast2 & - \\
\hline
\end{tabular}

${ }^{1)}$ Accessions PI and KU are from the National Genetic Resources Program, ARS, USDA and Plant Germplasm Institute, Kyoto University, Japan, respectively. ${ }^{2)}$ Accession provided by Dr. C. Konzak and maintained at Kanagawa University. ${ }^{3)}$ Accessions provided by the Kihara Institute for Biological Research, Yokohama, Japan and maintained at Kanagawa University. 
agarose gel electrophoresis. Inserts in 29 clones that showed the expected length (about 410-bp) were considered to be candidates of MULE transposase-coding sequences and subjected to the sequencing. Among 29 clones, 16 distinct sequences were recognized. According to the search performed in the NCBI, all clones were homologous to MULE transposase-coding sequences. These 16 DNA sequences were designated MoTU and were deposited in the DDBJ database (sequences AB354717 through AB354732).

\section{Classification of MoTUs}

MULEs of Triticum and Aegilops species were searched in the TIGR Wheat Genome Database. Twentynine MULE sequences significantly homologous to at least one of three maize elements (MuDR, Trap and Jittery) and four sugarcane MULEs (TE165, TE109, TE266 and
TE148), were found (Table 2). A phylogenetic analysis resulted in the classification of the MoTUs into five clades (Figure 1). MULEs of Triticum and Aegilops species were found in all but the Trap or Class II clade. Out of the 29 wheat MULEs identified by homology search, 19 MULEs were classified into the Jittery clade. All MoTUs were classified into a major $M u D R$ or Class I clade as expected, because degenerate primers were designed based on the $M u D R$ and $O s M u 4-2$ sequences. Furthermore, MoTUs were clearly divided into two subclasses: 11 of the 16 MoTUs belonged to subclass I and the remaining five belonged to subclass II. The average pair-wise identity between these two subclasses was $58.8 \%$ at the nucleotide sequence level. Subclass I exhibited the highest similarity to maize MURA transposase among MULE transposases identified in Triticum and Aegilops species. As shown in

Table 2 - MULE transposases detected in the genera Triticum and Aegilops.

\begin{tabular}{|c|c|c|c|c|}
\hline Name & Accession & Species & Comment & Reference \\
\hline MoTA-194 & BE431194 & T. aestivum & cDNA (EST) & Anderson et al. (unpublished data) \\
\hline MoTA-524 & BE497524 & T. aestivum & cDNA (EST) & Anderson et al. (unpublished data) \\
\hline MoTA-276 & BJ211276 & T. aestivum & cDNA (EST) & Kawaura et al. (2005) \\
\hline MoTA-746 & BJ260746 & T. aestivum & cDNA (EST) & Kawaura et al. (2005) \\
\hline MoTA-422 & BJ267422 & T. aestivum & cDNA (EST) & Kawaura et al. (2005) \\
\hline MoTA-307 & BJ271307 & T. aestivum & cDNA (EST) & Kawaura et al. (2005) \\
\hline MoTA-246 & BJ313246 & T. aestivum & cDNA (EST) & Kawaura et al. (2005) \\
\hline MoTA- 820 & BQ579820 & T. aestivum & cDNA (EST) & Anderson et al. (unpublished data) \\
\hline MoTA-942 & BU099942 & T. aestivum & cDNA (EST) & Anderson et al. (unpublished data) \\
\hline MoTA-723 & CA499723 & T. aestivum & cDNA (EST) & Anderson et al. (unpublished data) \\
\hline MoTA-252 & CA643252 & T. aestivum & cDNA (EST) & Tingey et al. (unpublished data) \\
\hline MoTA-186 & CD901186 & T. aestivum & cDNA (EST) & Genoplante $^{1)}$ (unpublished data) \\
\hline MoTA-055 & CD922055 & T. aestivum & cDNA (EST) & Genoplante (unpublished data) \\
\hline MoAT-136 & CG673136 & Ae. tauschii & genomic DNA & Li et al. (2004) \\
\hline MoAT-366 & CG674366 & Ae. tauschii & genomic DNA & Li et al. (2004) \\
\hline MoTA-126 & CJ632126 & T. aestivum & cDNA (EST) & Mochida et al. (2006) \\
\hline MoTA-928 & CJ630928 & T. aestivum & cDNA (EST) & Mochida et al. (2006) \\
\hline MoTA-852 & CJ637852 & T. aestivum & cDNA (EST) & Mochida et al. (2006) \\
\hline MoTA-210 & CJ642210 & T. aestivum & cDNA (EST) & Mochida et al. (2006) \\
\hline MoTA-280 & CJ659280 & T. aestivum & cDNA (EST) & Mochida et al. (2006) \\
\hline MoTA-333 & CJ668333 & T. aestivum & cDNA (EST) & Mochida et al. (2006) \\
\hline MoTA-244 & CJ714244 & T. aestivum & cDNA (EST) & MochidaK et al. (2006) \\
\hline MoTA-354 & CK156354 & T. aestivum & cDNA (EST) & Allard et al. (unpublished data) \\
\hline MoTA-630 & CK204630 & T. aestivum & cDNA (EST) & Allard et al. (unpublished data) \\
\hline MoTA-790 & CK210790 & T. aestivum & cDNA (EST) & Allard et al. (unpublished data) \\
\hline MoTA-641 & CV765641 & T. aestivum & cDNA (EST) & Allard et al. (unpublished data) \\
\hline MoTA-283 & DR735283 & T. aestivum & cDNA (EST) & Allard et al. (unpublished data) \\
\hline MoTA-290 & DR735290 & T. aestivum & cDNA (EST) & Allard et al. (unpublished data) \\
\hline MoTD-831 & TREP8312) & T. durum & genomic DNA & Wicker et al. (2003) \\
\hline
\end{tabular}

\footnotetext{
${ }^{1)}$ The French Plant Genomics program, ${ }^{2)}$ Accession number from the Triticeae Repeat Sequence Database (Wicker et al., 2002).
} 
Figure 1, the sequence diversity of subclass I was larger than that of subclass II.

\section{Distribution of MULEs in wheat}

Distribution of MULEs belonging to subclass I and II was surveyed in the 28 accessions of Triticum and Aegilops listed in Table 1 by DNA gel blot analysis. We used

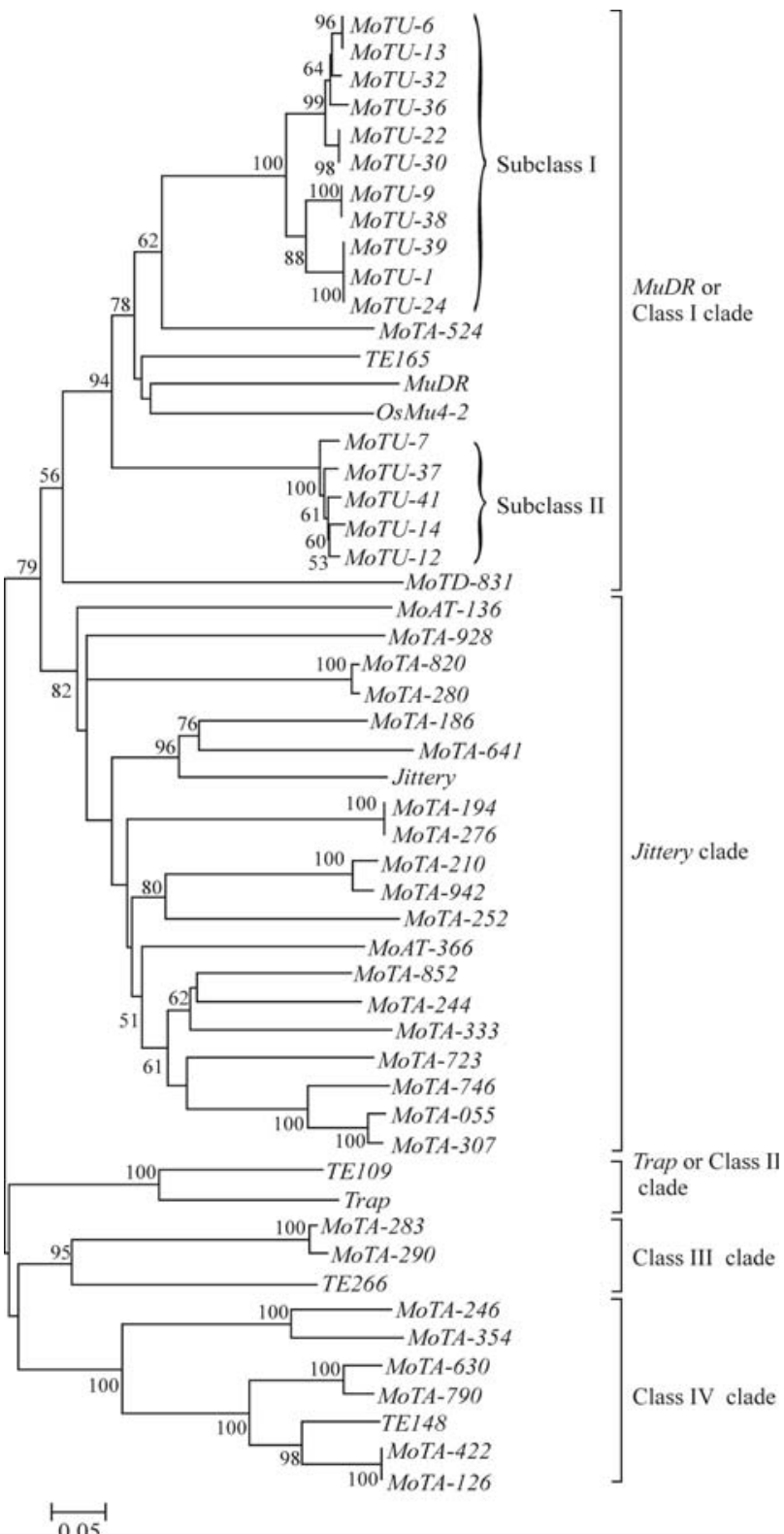

Figure 1 - Phylogenetic analysis of the nucleotide sequences of MULE transposases from Triticum and Aegilops species. Coding sequences corresponding to amino acids 385 through 477 of the maize MURA were used for the analysis. A phylogenetic tree was constructed by the NJ method. Bootstrap values higher than $50 \%$ for 1000 replications are shown at the nodes. Classification into classes followed Rossi et al. (2004). MuDR, Jittery and Trap are from maize and OsMu4-2 (Asakura et al. 2002) is from rice. Sequences denoted with TE are from sugarcane (Rossi et al. 2004) and sequences designated MoTU were isolated from $T$. urartu. Details of other sequences identified in the genera Triticum and Aegilops by blastn and tblastx searches are described in Table 2 .
MoTU-32 and MoTU-12 as probes because they represent two MoTU subclasses. The hybridization patterns with each probe clearly differed in these accessions. Several bands were detected with the MoTU-32 probe (subclass I) in the diploids with the $\mathrm{A}, \mathrm{S}, \mathrm{S}^{1}$ and $\mathrm{D}$ genomes and in the two types of timopheevi wheat, $T$. araraticum and $T$. timopheevi, with the AG genome (Figure 2A). A few more bands were detected in the emmer wheat (T. dicoccoides, $T$. dicoccum and $T$. durum) with the $\mathrm{AB}$ genome and in the common wheat (T. spelta and T. aestivum) with the ABD

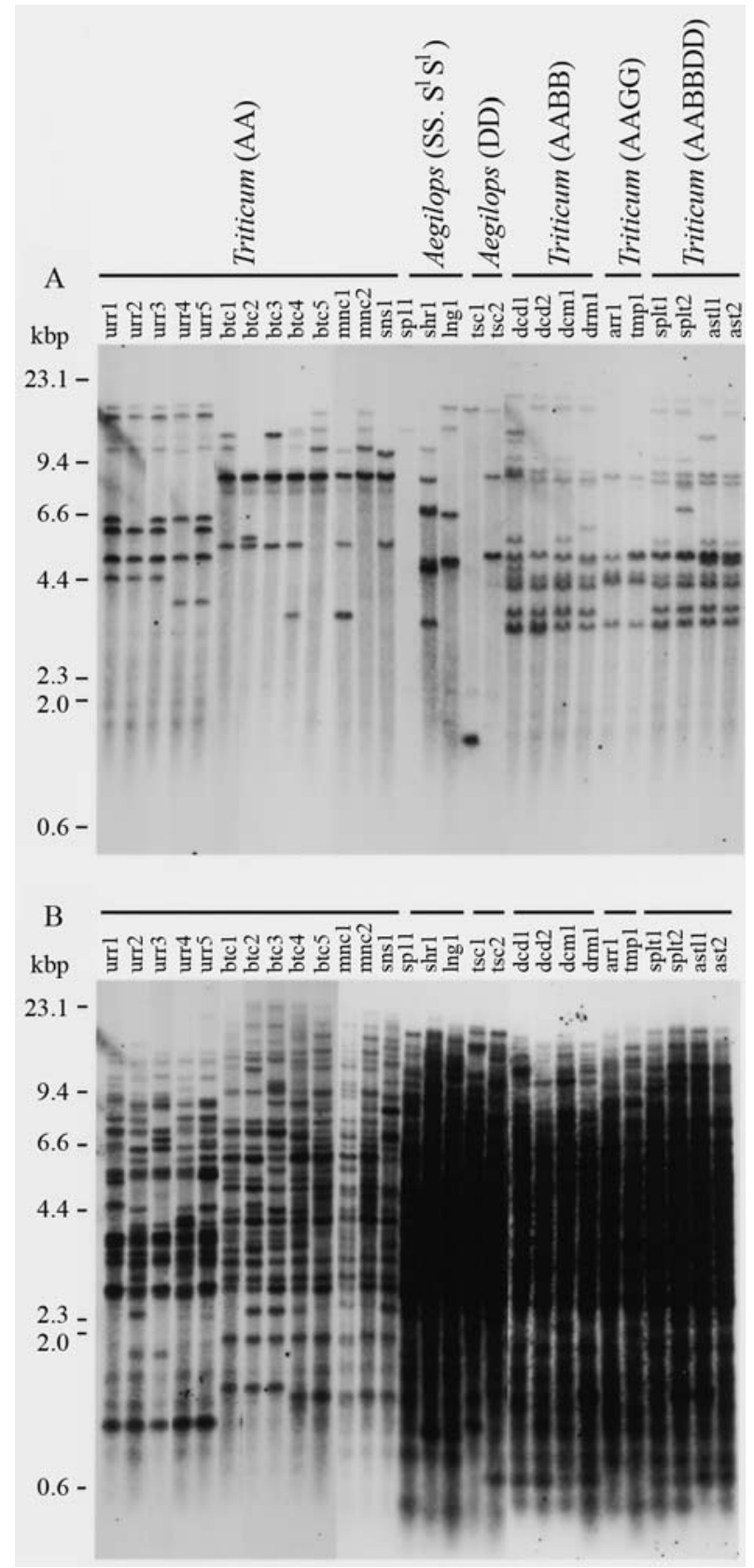

Figure 2 - DNA blot analysis of MULE transposases in wheat including Triticum and Aegilops species. Abbreviations of the accessions are shown in Table 1. DNA digested with Hind III was hybridized with the probes: (A) MoTU-32 (subclass I) and (B) MoTU-12 (subclass II). 
genome. On the other hand, MoTU-12 (subclass II) detected more than 15 major bands in the einkorn wheat (T. urartu, T. boeoticum and T. monococcum) with the A genome and many more bands in the Aegilops species with the $\mathrm{S}, \mathrm{S}^{1}$ or D genomes (Figure 2B). Numerous bands were also detected in all of the tetraploid and hexaploid wheat accessions. Subclass II MULEs therefore represented a highcopy element in the genomes of Triticum and Aegilops species, particularly in the $\mathrm{S}, \mathrm{S}^{1}$ and $\mathrm{D}$ genomes and probably also in the B and $\mathrm{G}$ genomes. In contrast to subclass II MULEs, subclass I MULEs existed as a low-copy element.

\section{Distribution of MULEs among some grass species}

The distribution of MULEs homologous to the two subclasses was studied through DNA gel blot analysis in 16 accessions from six grass species. Intense and distinct hybridization bands of subclass I were detected by MoTU-32 in all accessions of barley and rye, which belong to the tribe Triticeae (Figure 3A). More bands were detected in barley than in rye and diploid wheat (Figure 2A). Weak signals were detected in rice and no signals were detected in oat, maize and sorghum. Intense hybridization bands of subclass II were detected by MoTU-12 in all accessions of barley, rye and oat, which belong to the subfamily Pooideae (Figure 3B). In barley and rye, like in wheat, numerous bands were detected. In oat, however, two intense signals, with $3.6-\mathrm{kbp}$ and $1.3-\mathrm{kbp}$, were detected. The 1.3-kbp signal was extremely intense, indicating that the subclass II transposase existed as a high copy number element also in oat. No subclass II signals were detected in rice, maize, and sorghum.

\section{Discussion}

\section{Sequence differentiation of MULE transposases in wheat}

MULE transposase homologs found in Triticum and Aegilops were classified into five clades, i.e., $M u D R$ or Class I, Jittery, Trap or Class II, Class III and Class IV (Figure 1). Most of MULE transposases in plants were reported to exhibit higher similarity to the transposase of Jittery than to MURA (Walbot and Rudenko 2002; Xu et al. 2004) and the Jittery clade also included the largest numbers of MULEs in T. aestivum. Many MULE transposases belonging to the Jittery clade are probably also present in $T$. urartu. MoTUs, however, showed a higher similarity to the transposase of $M u D R$ than to that of Jittery. This result was most probably caused by a PCR bias based on sequence differences between $M u D R$ and Jittery at the target regions of primers. Consequently, we selectively amplified MULE transposase sequences belonging to the $M u D R$ clade.

Clear sequence differentiation of MULEs was found in the MuDR clade. MoTUs consisted of two distinct subclasses that exhibited an average pair-wise identity of $58.8 \%$ at the nucleotide sequence level. Among wheat

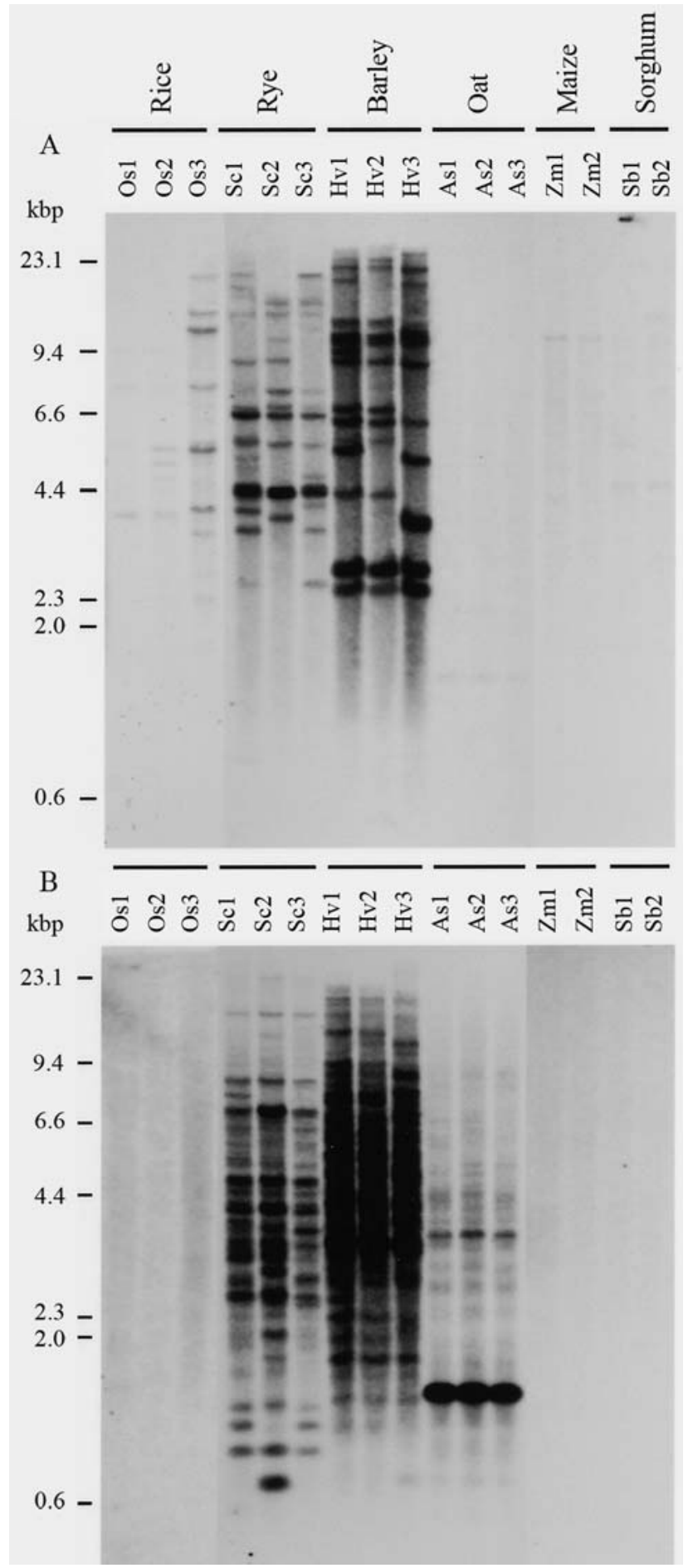

Figure 3 - DNA blot analysis of MULE transposases in six grass species. Abbreviations of the accessions are as follows: Os1 - Oryza sativa ssp. japonica cv. Nipponbare; Os2 - ssp. javanica accession no. 242 (National Institute of Genetics (NIG), Japan); Os3 - ssp. indica accession n. 101 (NIG); Sc1 - Secale cereale cv. Haruichiban; Sc2 - cv. King II; Sc3 - cv. Steel; Hv1 - Hordium vulgare cv. Ingrid; Hv2 - cv. Betzes; Hv3 - cv. Suwon6; As1 - Avena sativa cv. Brooks; As2 - cv. Kanota; As3 - cv. Ogle; Zm1 - Zea mayz cv. Woody corn (Sakata Seed corporation (SSC), Japan); Zm2 - cv. Pop corn (SSC); Sb1 - Sorghum bicolor accession no. PI 244057 (National Genetic Resources Program, ARS (NGRP), USDA); Sb2 - accession no. NSL 92562 (NGRP). DNA digested with Hind III was hybridized with probes: (A) MoTU-32 (subclass I) and (B) MoTU-12 (subclass II). 
MULEs, subclass I was the closest to the maize MURA. Sequence diversity was higher in subclass I than in subclass II MULEs. The differentiation within subclass I may have occurred earlier than that within subclass II.

\section{Copy number variation of MULEs in wheat}

DNA gel blot analysis showed that subclass II MULEs existed as a high-copy number element in the genomes of wheat, rye and barley (Figures 2B and 3B). Furthermore, it is intriguing that the copy number of subclass II transposases obviously differed among the ancestral diploid genomes: the genomes S, $\mathrm{S}^{1}$ and D of Aegilops species possessed higher copy numbers than the A genome of diploid Triticum species. Tetraploid and hexaploid wheat genomes also contained numerous subclass II transposases. The B genome of emmer wheat and common wheat and the G genome of timopheevi wheat were most probably derived from the $\mathrm{S}$ genome of Aegilops speltoides (Dvorak and Zhang 1990). Aegilops tauschii donated the D genome to common wheat (Kihara 1944; McFadden and Sears 1946). Tetraploid and hexaploid wheat thus probably inherited the numerous copies of subclass II MULEs through their evolution by allopolyploidization. This copy number variation among diploid species probably reflects historical differences in transposition frequencies of subclass II MULEs after the differentiation of the genera Triticum and Aegilops. The factors determining such copy number variation require further studies.

The copy number of subclass II MULEs was much higher than that of subclass I in wheat, rye and barley (Figures $2 \mathrm{~A}$ and $3 \mathrm{~A}$ ). Sequence diversity of subclass II was lower than that of subclass I. These results suggest that rapid amplification of subclass II MULEs has recently occurred. Furthermore, the results also suggest that the transposition of each MULE subclass is under a different regulation. The MURA transposase binding site (MBS), a 32-bp motif in the TIRs, is well conserved among the mobile Mutator elements (Benito and Walbot 1997; Rudenko and Walbot 2001). It was suggested that transposase active for transposition of subclass II MoTUs might not be able to recognize MBSs of subclass I MoTUs. A similar behavior was observed between distinct groups of mariner-like elements coexisting in a Drosophila genome (Lohe et al. 1995).

\section{MULE dynamics in grass species}

DNA gel blot analysis revealed that MULEs of the two subclasses were present at least within the tribe Triticeae (Figure 3). This result suggests that the evolution of these subclasses occurred before or immediately after the establishment of the tribe Triticeae. However, a clear differentiation exists between the two subclasses. Subclass I MULEs were found in rice of the subfamily Ehrhartoideae but not in oat of the tribe Aveneae that belongs to the subfamily Pooideae. Oat, on the other hand, possessed sub- class II MULEs (Figure 3B). This could be explained by the stochastic loss of subclass I MULEs in oat, as originally proposed to account for the patchy distribution of $P$ elements among Drosophila melanogaster strains (Engels 1981). More extensive studies are required in order to clarify the distribution and sequence diversity of these two subclasses of MULEs and to understand MULE dynamics in grass species.

\section{Acknowledgments}

We thank Dr. J. Fujigaki, Junior College of Tokyo University of Agriculture, Japan, for providing us with rye seeds and Dr. T. Ishii, Kobe University, Japan, for rice DNAs. We are also grateful to the National Genetic Resources Program, ARS, USDA, for seeds of barley, oat and sorghum.

\section{References}

Altschul SF, Madden TL, Schaffer AA, Zang J, Zhang Z, Miller W and Lipman DJ (1997) Gapped BLAST and PSI-BLAST: A new generation of protein database search programs. Nucleic Acids Res 25:3389-3402.

Asakura N, Nakamura C, Ishii T, Kasai Y and Yoshida S (2002) A transcriptionally active maize $M u D R$-like transposable element in rice and its relatives. Mol Genet Genomics 268:321-330.

Benito MI and Walbot V (1997) Characterization of the maize Mutator transposable element MURA transposase as a DNA-binding protein. Mol Cell Biol 17:5156-5175.

Bennetzen JL (1996) The Mutator transposable element system of maize. In: Saedler H and Gierl A (eds) Transposable Elements. Springer-Verlag, New York, pp 195-229.

Chantret N, Salse J, Sabot F, Rahman S, Bellec A, Laubin B, Dubois I, Dossat C, Sourdille P, Joudrier P, et al. 2005) Molecular basis of evolutionary events that shaped the hardness locus in diploid and polyploid wheat species (Triticum and Aegilops). Plant Cell 17:1033-1045.

Chalvet F, Grimaldi C, Kaper F, Langin T and Daboussi MJ (2003) Hop, an active Mutator-like element in the genome of the fungus Fusarium oxysporum. Mol Biol Evol 20:1362-1375.

Comelli P, Konig J and Werr W (1999) Alternative splicing of two leading exons partitions promoter activity between the coding regions of the maize homeobox gene Zmhoxla and Trap (transposon-associated protein). Plant Mol Biol 41:615-625.

Dvorak J and Zhang H-B (1990) ariation in repeated nucleotide sequences sheds light on the phylogeny of the wheat B and $\mathrm{G}$ genomes. Proc Natl Acad Sci USA 87:9640-9644.

Eisen J, Benito MI and Walbot V (1994) Sequence similarity of putative transposase links the maize Mutator autonomous element and a group of bacterial insertion sequences. $\mathrm{Nu}-$ cleic Acids Res 22:2634-2636.

Engels WR (1981) Hybrid dysgenesis in Drosophila and the stochastic loss hypothesis. Cold Spring Harbor Symp Quant Biol 45:561-565.

Feldman M, Lupton FGH and Miller TE (1995) Wheats, Triticum spp. (Gramineae-Triticinae). In: Smartt J and Simmonds 
NW (eds) Evolution of Crop Plants. Longman Scientific and Technical, Harlow, pp 184-192.

Kawaura K, Mochida K and Ogihara Y (2005) Expression profile of two storage-protein gene families in hexaploid wheat revealed by large-scale analysis of expressed sequence tags. Plant Physiol 139:1870-1880.

Kihara H (1944) Discovery of the DD-analyzer, one of the ancestors of Triticum vulgare - in Japanese. Agric Hort Tokyo 19:889-890.

Kihara H and Tanaka M (1970) Addendum to the classification of the genus Aegilops by means of genome-analysis. Wheat Inf Serv 30:1-2.

Kimber G and Sears ER (1983) Assignment of genome symbols in the Triticeae. In: Sakamoto S (ed) Proceedings of the 6th Int. Wheat Genet Symp Plant Germ-Plasm Institute. Kyoto University, Kyoto, pp 1195-1196.

Kumar S, Tamura K and Nei M (2004) MEGA 3: Integrated software for molecular evolutionary genetics Analysis and sequence alignment. Brief Bioinformat 5:150-163.

Li W, Zhang P, Fellers JP, Friebe B and Gill BS (2004) Sequence composition, organization, and evolution of the core Triticeae genome. Plant J 40:500-511.

Lisch D (2002) Mutator transposons. Trends Plant Sci 7:498-504.

Lisch D, Chomet P and Freeling M (1995) Genetic characterization of the Mutator system in maize: Behavior and regulation of $M u$ transposons in a minimal line. Genetics 139:1777-1796.

Lisch DR, Freeling M, Langham RJ and Choy MY (2001) Mutator transposase is widespread in the grasses. Plant Physiol 125:1293-1303.

Lisch DR, Girard L, Donlin M and Freeling M (1999) Functional analysis of deletion derivatives of the maize transposon $M u D R$ delineates roles for the MURA and MURB proteins. Genetics 151:331-341.

Lohe AR, Moriyama EN, Lidholm D-A and Hartl DL (1995) Horizontal transmission, vertical inactivation, and stochastic loss of mariner-like transposable elements. Mol Biol Evol 12:62-72.

Mao L, Wood TC, Yu Y, Budiman MA, Tomkins J, Woo S, Sasinowski M, Presting G, Frisch D, Goff S, et al. (2000) Rice transposable elements: A survey of 73,000 sequencetagged-connectors. Genome Res 10:982-990.

Marchler-Bauer A and Bryant SH (2004) CD-Search: Protein domain annotations onthe fly. Nucleic Acids Res 32:W327331.

McFadden ES and Sears ER (1946) he origin of Triticum spelta and its free-threshing hexaploid relatives. J Hered 37:81-89.

Mochida K, Kawaura K, Shimosaka E, Kawakami N, Shin-I T, Kohara Y, Yamazaki Y and Ogihara Y (2006) Tissue expression map of a large number of expressed sequence tags and its application to in silico screening of stress response genes in common wheat. Mol Genet Genomics 276:304312.

Raizada MN, Nan GL and Walbot V (2001) Somatic and germinal mobility of the RescueMu transposon in transgenic maize. Plant Cell 13:1587-1608.
Raizada MN and Walbot V (2000) The late developmental pattern of $M u$ transposon excision is conferred by a CaMV 35Sdriven MURA cDNA in transgenic maize. Plant Cell 12:5-21.

Robertson DS (1978) Characterization of a Mutator system in maize. Mutat Res 51:21-28.

Rossi M, Araujo PG, de Jesus EM, Varani AM and Van Sluys MA (2004) Comparative analysis of Mutator-like transposases in sugarcane. Mol Genet Genomics 272:194-203.

Rossi M, Araujo PG and Van Sluys MA (2001) Survey of transposable elements in sugarcane expressed sequence tags (ESTs). Genet Mol Biol 24:147-154.

Rudenko GN and Walbot V (2001) Expression and post-transcriptional regulation of maize transposable element $M u D R$ and its derivatives. Plant Cell 13:553-570.

Saitou N and Nei M (1987) The neighbor-joining method: A new method for reconstructing phylogenetic trees. Mol Biol Evol 4:406-425.

Thompson JD, Gibson TJ, Plewniak F, Jeanmougin F and Higgins DG (1997) The CLUSTAL X windows interface: Flexible strategies for multiple sequence alignment aided by quality analysis tools. Nucleic Acids Res 25:4876-4882.

Tsunewaki K and Nakamura H (1995) Genomic relationships and phylogeny in wheat obtained by RFLP analysis of nuclear DNA. In: Tsunewaki K (ed) Plant Genome and Plastome. Their Structure and Evolution. Kodansha, Tokyo, pp 115-127.

Walbot V and Rudenko GN (2002) MuDR/Mu transposable elements of maize. In: Craig NL, Craigie R, Gellert M and Lambowitz AM (eds) Mobile DNA II. ASM Press, Washington DC, pp 533-564.

Wicker T, Matthews DE and Keller B (2002) TREP: A database for Triticeae repetitive elements. Trends Plant Sci 7:561562.

Wicker T, Yahiaoui N, Guyot R, Schlagenhauf E, Liu ZD, Dubcovsky J and Keller B (2003) Rapid genome divergence at orthologous low molecular weight glutenin loci of the A and $\mathrm{A}(\mathrm{m})$ genomes of wheat. Plant Cell 15:1186-1197.

Xu Z, Yan X, Maurais S, Fu H, O'Brien DG, Mottinger J and Dooner HK (2004) Jittery, a Mutator distant relative with a paradoxical mobile behavior: Excision without reinsertion. Plant Cell 16:1105-1114.

Yan L, Echenique V, Busso C, SanMiguel P, Ramakrishna W, Bennetzen JL, Harrington S and Dubcovsky J (2002) Cereal genes similar to $\operatorname{Snf} 2$ define a new subfamily that includes human and mouse genes. Mol Genet Genomics 268:488499.

Yoshida S, Tamaki K, Watanabe K, Fujino M and Nakamura C (1998) A maize $M u D R$-like element expressed in rice callus subcultured with proline. Hereditas 129:95-99.

Yu Z, Wright SI and Bureau TE (2000) Mutator-like elements in Arabidopsis thaliana: Structure, diversity and evolution. Genetics 156:2019-2031.

Associate Editor: Márcio de Castro Silva Filho

License information: This is an open-access article distributed under the terms of the Creative Commons Attribution License, which permits unrestricted use, distribution, and reproduction in any medium, provided the original work is properly cited. 